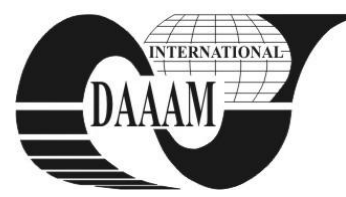

\title{
VISION BASED CONTROL ALGORITHM FOR A MOBILE MANIPULATOR
}

\author{
CETIN, L[event]; BASER, O[zgun]; KESKIN, O[nur] \& UYAR, E[rol]
}

\begin{abstract}
An autonomous mobile manipulator, a wheeled mobile platform carrying a manipulator arm, with a vision based target acquisition system can be able not only to find and maintain fixation on a moving target but also to manipulate or handle it while the system itself is in motion Using vision based control methodology, two different control types for mobile platform and manipulator is applied. Mobile platform is controlled with a hybrid visual servo controller.. Manipulator motion is controlled via dynamic look and move control strategy, in which actuator references updated instantaneously according to acquired target position. Control of the mobile manipulator is done by task sequencing. A task is separated in to two parts: Results for given control methodology are demonstrated final system succeeds visually controlled positioning tasks, successfully.
\end{abstract}

Key words: visual servoing, mobile manipulator)

\section{INTRODUCTION}

A mobile manipulator consists of an articulated arm mounted on a mobile platform. Since this mechanical arrangement combines the dexterity of the former with the workspace extension of the latter, it is clearly appealing for many applications (Khatib et al. 1996). The locomotion of the platform is typically obtained through wheels. Therefore analysis and control of the mobile robots are parallel to developments in wheeled mobile robot research.

Vision based control is a technique where the appearance of a target in the image or an estimation of the observed target position is used to control the position of the robot and to move it to a desired position in the 3D scene. (Bloch et al. 2000, Hutchinson 1996 , Hashimoto 2003).

In this study control of non holonomic mobile manipulator is done by task sequencing. Any positioning task is realized in two stages: Mobile platform moves till target is placed in manipulator workspace and in proper orientation that gripper (tracking) can hold, afterwards manipulator moves to hold target (handling).This distribution reduces the control problem in to two sub problems: control of the mobile platform and control of manipulator and controllers described above can be implemented

\section{PROBLEM DEFINITION}

In very general terms, for exploiting the interaction between perceptions and actions, it is necessary to model system and calibrates different systems to act in cascaded operations. Using imaging models developed for cameras and priori known patterns, it is possible to fully parameterize the image point and world point relation. For binocular stereo applications which fully imitates human eye configuration, it is possible to find 3D coordinates of a monitored point with calibrated setup.
Implementation of binocular stereo rig applications in robotic systems, sensor data can be converted to task frame data and can be exploited in standard kinematics models.

Constructing a 3-dimensional structural representation involves finding pairs of pixels from the two images which correspond to the same point in space. Given the disparity map and the geometry of the stereo setup, triangulation is the task of computing the 3D position of points in the images (Trucco \& Verri 1994).

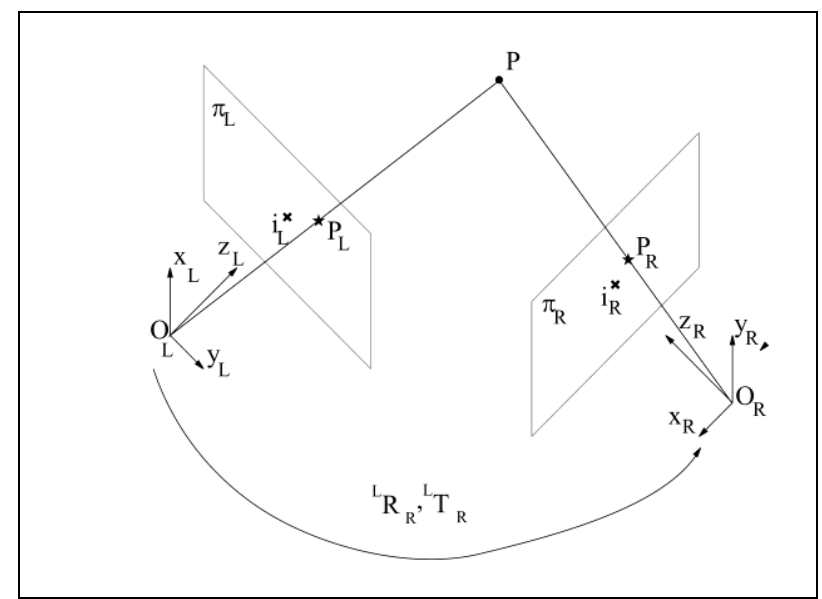

Fig. 1. Stereo camera pair and relations between coordinate frames.

Vectors $\mathrm{P}_{\mathrm{L}}$ and $\mathrm{P}_{\mathrm{R}}$ are related to each other with rigid body motion equations if rotation matrix $\mathrm{R}$ and translation between cameras coordinate frames are known.

$$
P_{L}={ }_{L} R^{R} P_{R}+{ }_{L} T^{R}
$$

\section{CONTROL OF MOBILE MANIPULATOR}

In proposed system, robot ability is not limited with tracking or manipulation ability as it is separated in active vision and visual servoing applications but a fusion of the capabilities from two research field. Concerning structure of implementation model, three problems are occurred to be solved:

1. Control of mobile platform: It is necessary to track targets in the field of vision and in the reach limitations of the robot manipulator. This problem, by its nature, is a visual servoing problem where pose error cannot be directly related to sensory and actuators signals but related to integral of them. Cameras mounted on a mobile platform are not only track objects to keep them in the field of view of cameras but also keep the object within dexterous workspace of the planar manipulator. Tracking ability of active vision system, namely 
cameras on mobile platform, is constrained with operation plane of the planar manipulator.

$$
\begin{gathered}
\Delta w=\left\{\begin{array}{cc}
\Delta w_{\max } & \frac{C_{T}-C_{r}}{\text { width }}<-1 \\
\frac{C_{T}-C_{r}}{\text { width }} * \Delta w_{\max } & 1 \geq \frac{C_{T}-C_{r}}{\text { width }} \geq-1 \\
\Delta w_{\max } & \frac{C_{T}-C_{r}}{\text { width }}>1
\end{array}\right. \\
w=\left\{\begin{array}{cc}
\frac{d_{T}-d_{r}}{\text { width }} * w_{\text {max }} & \frac{d_{T}-d_{r}}{\text { width }} \leq 1 \\
w_{\text {max }} & \frac{d_{T}-d_{r}}{\text { width }}>1
\end{array}\right.
\end{gathered}
$$

2. Control of robot manipulator: Planar manipulator is to track position of the target in a perpendicular plane which forms 3D world when combined with plane that mobile platform moves. Control task is to dynamically handle target, viewed by cameras. Dynamic look and move paradigm is suitable for the general case in this study, because the system equipped with servo controllers.

$$
\begin{aligned}
& x^{2}+y^{2}=l_{1}^{2}+l_{2}^{2}-l_{1} l_{2} \cos \left(\pi-\theta_{2}\right) \\
& \theta_{2}=\mp a \cos \left(\frac{x^{2}+y^{2}-l_{1}^{2}-l_{2}^{2}}{l_{1} l_{2}}\right) \\
& \theta_{1}=\operatorname{atan}\left(\frac{y}{x}\right)-\operatorname{atan}\left(\frac{l_{2} \sin \theta_{2}}{l_{1}+l_{2} \cos \theta_{2}},\right.
\end{aligned}
$$

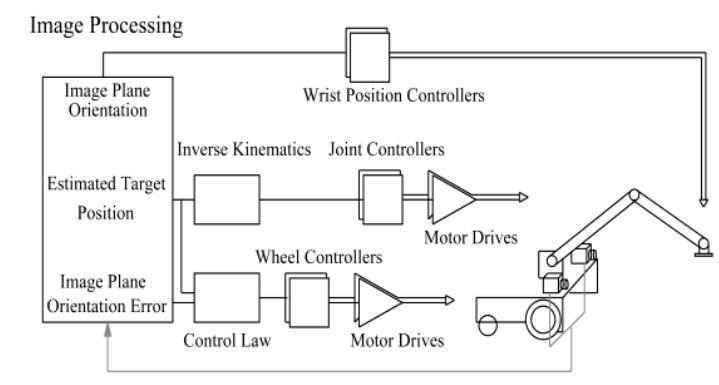

Fig 2. Vision system integration to mobile robot

\section{EXPERIMENTAL RESULTS}

In following results of experiments for described algorithms are presented (Figure 7).Task defined for this study is picking up a target object via visual feedback in task space. Target object used in experiments is a LED torch. Expected motion of the robotic system is execution of following tasks, sequentially. Actuators are equipped with PI controllers to follow commanded values from vision based control algorithm. Results show that mobile manipulator can perform positioning actions following visual observations through stereo camera calibration setup

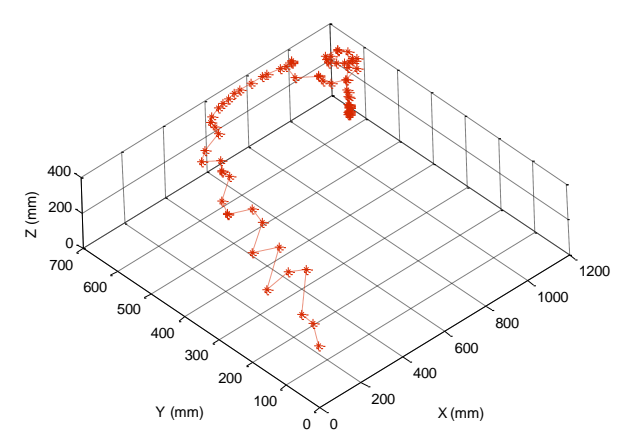

(a) End effector trajectory

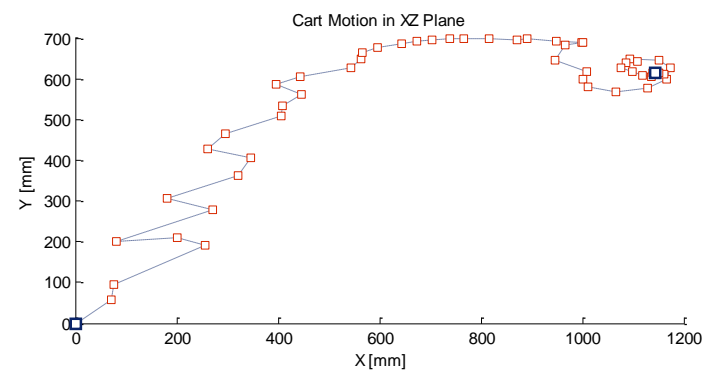

(b) Mobile Platform trajectory

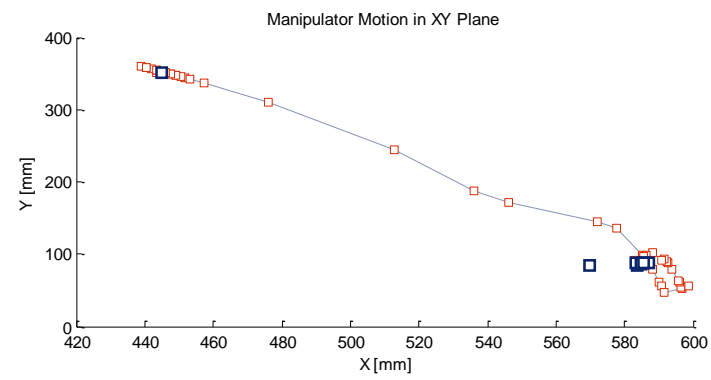

(c) Manipulator end effector trajectory

Fig 3. Mobile Manipulator Control Experiment (-- reference, $x$ actual) and control signal (-)

\section{CONCLUSIONS}

In this study, computer and vision systems are integrated as a part of mobile manipulator control process. Using developed kinematical decomposition, design procedure starts with mobile platform controller. Using image and position based control methodologies; control of the mobile platform is succeeded to fulfill the task keeping the manipulator gripper in the workspace of the manipulator and target in the same vertical frame with gripper. Manipulator controller is operated in a dynamic look and move procedure where joint references instantaneously updated.

\section{REFERENCES}

Hashimoto, K. , 2003 "A review on vision-based control of robot manipulators". Advanced Robotics vol.17(10), 969990

Hutchinson, S.A., Hager, , 1996 G.D. \& Corke P.I. "A tutorial on visual servo control". IEEE Transactions on robotics and. automation, vol 12(5), 651-670

Khatib, O., Yokoi, K. Chang, K., Ruspini, D., Holmberg R. \& Casal,A. , 1996 "Coordination and Decentralized Cooperation of Multiple Mobile Manipulator". Journal of Robotic Systems vol 13(11), pp 755-764

Trucco, E. \& Verri, A, 1998.. Introductory techniques for 3-d computer vision. New Jersey: Prentice-Hall 